\title{
Clinical and Laboratory Characteristics of Patients with Peritoneal Tuberculosis Mimicking Advanced Ovarian Cancer
}

\author{
Amita Maheshwari ${ }^{1}$ Sudeep Gupta ${ }^{2, \odot ~ S h w e t a ~ R a i ~}{ }^{1}$ Bharat Rekhi ${ }^{3}$ Rohini Kelkar ${ }^{4}$ TS Shylasree ${ }^{1}$ \\ Santosh Menon ${ }^{3}$ Kedar Deodhar ${ }^{3} \quad$ Meenakshi Thakur $^{5} \quad$ Ushasree Das $^{1} \quad$ Stuti Gupta ${ }^{1}$ Sandeep Tandon ${ }^{6}$ \\ ${ }^{1}$ Department of Gynecologic Oncology, Tata Memorial Centre and \\ Homi Bhabha National Institute, Mumbai, Maharashtra, India \\ ${ }^{2}$ Deptartment of Medical Oncology, Tata Memorial Centre and \\ Homi Bhabha National Institute, Mumbai, Maharashtra, India \\ Address for correspondence Amita Maheshwari, MD, Department \\ of Gynecologic Oncology, Tata Memorial Centre and Homi Bhabha \\ National Institute, Dr. Ernest Borges Road, Parel, Mumbai 400012, \\ India (e-mail: maheshwariamita@yahoo.com).
}

${ }^{3}$ Deptartment of Pathology, Tata Memorial Centre and Homi Bhabha National Institute, Mumbai, Maharashtra, India

${ }^{4}$ Deptartment of Microbiology, Tata Memorial Centre and Homi Bhabha National Institute, Mumbai, Maharashtra, India

${ }^{5}$ Deptartment of Radiodiagnosis, Tata Memorial Centre and Homi Bhabha National Institute, Mumbai, Maharashtra, India

${ }^{6}$ Department of Medicine, Tata Memorial Centre and Homi Bhabha National Institute, Mumbai, Maharashtra, India

South Asian J Cancer 2021;10:102-106.

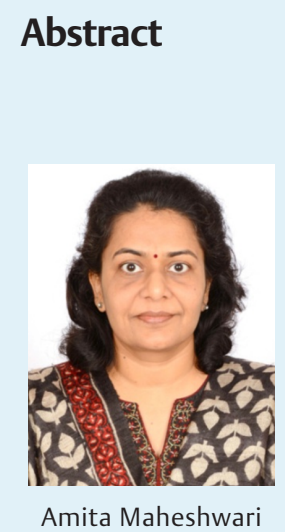

Keywords

- abdominal tuberculosis

- ovarian cancer

- primary peritoneal cancer
Objectives Peritoneal tuberculosis can mimic advanced abdominal malignancy. We describe clinical and laboratory characteristics in a series of female patients with peritoneal tuberculosis who were referred to a tertiary cancer center with a diagnosis of suspected advanced ovarian/primary peritoneal cancer.

Materials and Methods Details of clinical features, laboratory results including serum tumor markers, radiological findings, and ascitic fluid evaluation were retrospectively collected from hospital records for patients diagnosed to have peritoneal tuberculosis and reported descriptively.

Statistical Analysis Descriptive statistics was performed using SPSS Statistics for Windows software, version 20.0 (SPSS, Chicago, Illinois).

Results Between January 2009 and December 2017, 120 patients of peritoneal tuberculosis with a median age 41 years (range, 15-79 years) were identified. Of these 112 (93.3\%; 95\% Cl 88.9-97.8\%) patients had ascites and 63 (52.5\%; 95\% Cl 43.6-61.4\%) had adnexal mass at presentation. Mean serum cancer antigen 125 (CA-125) level was 666.9 (range, 38-18,554) $\mathrm{U} / \mathrm{mL}$. Ascitic fluid was negative for malignant cells in all patients and lymphocyte rich exudate was seen in 103 (91.9\%; 86.9-97.0\%) patients. Ascitic fluid adenosine deaminase (ADA) level was more than $40 \mathrm{U} / \mathrm{L}$ in 107 (95.5\%; 95\% Cl 91.7-99.4\%). Ascitic fluid Ziel-Neelsen staining was positive in $4 / 62$ (6.5\%; 95\% Cl 0.3-12.6\%) patients while ascitic fluid culture examination for mycobacterium tuberculosis was positive in $7 / 59$ (11.9\%; 95\% Cl 3.6-20.1\%) patients. The diagnosis of tuberculosis was based on image-guided biopsy in 44 (36.7\%) patients, surgical biopsy in 8 (6.7\%) patients, and a combination of clinicoradiological and laboratory features in 68 (56.7\%) patients. All patients received standard antitubercular treatment.

Conclusions The study results suggest that peritoneal tuberculosis has clinical, radiological, and serological profile which may mimic advanced ovarian/primary peritoneal cancer. Peritoneal tuberculosis should be considered in the differential diagnosis of advanced abdominal malignancy.
DOI https://doi.org/10.1055/s-0041-1736030 ISSN 2278-330X

How to cite this article: Maheshwari A, Gupta S, Rai S, Rekhi B, Kelkar R, Shylasree TS, Menon S, Deodhar K, Thakur M, Das U, Gupta S, Tandon S. Clinical and Laboratory Characteristics of Patients with Peritoneal Tuberculosis Mimicking Advanced Ovarian Cancer. South Asian J Cancer 2021;10(2):102-106. (c) 2021. Medlntel Services Pvt Ltd.

This is an open access article published by Thieme under the terms of the Creative Commons Attribution-NonDerivative-NonCommercial-License, permitting copying and reproduction so long as the original work is given appropriate credit. Contents may not be used for commercial purposes, or adapted, remixed, transformed or built upon. (https://creativecommons.org/licenses/by-nc-nd/4.0/).

Thieme Medical and Scientific Publishers Private Ltd A-12, Second Floor, Sector -2, NOIDA -201301, India 


\section{Introduction}

Peritoneal tuberculosis is characterized by varying involvement of visceral and parietal peritonii, omentum, spleen, lymph nodes, and female genital tract. Other types of abdominal tuberculosis include intestinal and hepatic involvement. ${ }^{1}$ Peritoneal tuberculosis occurs either from hematogenous spread, from a primary pulmonary infection, or from the reactivation of latent foci in the peritoneum. Common clinical, imaging, and laboratory features of peritoneal tuberculosis in females include pelvic pain or discomfort, ascites, disseminated peritoneal involvement, adnexal masses, and elevated serum CA-125.These features overlap substantially with the clinical presentation of advanced ovarian carcinoma (OC) or primary peritoneal carcinoma (PPC) leading to a misdiagnosis of advanced malignancy. ${ }^{1-7}$ Conversely, in tuberculosis endemic countries, patients with malignancy may be inappropriately started on antitubercular treatment leading to delay in cancer management. Considering entirely different treatment and outcomes, it is important to differentiate these conditions early in their disease course.

Herein, we report the clinical profile and management of patients with peritoneal tuberculosis who were referred to our cancer center with a provisional diagnosis of advanced ovarian or peritoneal cancer.

\section{Materials and Methods}

This analysis was undertaken after obtaining approval from institutional ethics committee. The data of all female patients who were diagnosed with peritoneal tuberculosis during the study period were retrospectively collected from the electronic medical records and case files. All patients were referred to the gynecologic oncology department of our hospital with a suspicion of advanced ovarian or primary peritoneal malignancy. The collected data included clinical characteristics, laboratory results, radiological findings, diagnostic modalities, treatment, and outcomes. The clinical features included age, symptoms, menstruation history, personal or family history of tuberculosis, medical comorbidities, past abdominal surgery, and physical examination findings. Laboratory data included complete blood count, serum biochemistry, serum CA-125, CA19.9, and carcino embryonic antigen (CEA) levels. Ascitic fluid analysis included cytology, acid-fast bacilli (AFB) smear, AFB culture, and adenosine deaminase (ADA) level. Radiological examination included chest radiograph; contrast-enhanced computed tomography (CECT) of thorax, abdomen, and pelvis and/or PET/CT scan. Details of image-guided biopsy and diagnostic surgical intervention, whenever performed, were collected.

The diagnosis of peritoneal tuberculosis was established by either histopathology, ascitic fluid microbiology, or a combination of clinical, radiological, and laboratory findings, after exclusion of malignancy. In the latter group, an empirical course of antitubercular drugs was administered with close clinical, serological, and radiological monitoring of response to treatment. All patients received standard tuberculosis treatment with isoniazid, rifampicin, pyrazinamide, and ethambutol for a duration of 9 months.

\section{Results}

Between January 2009 and December 2017, 120 patients with a diagnosis of peritoneal tuberculosis were identified from institutional records, with median age of 41 (range, 15-79) years. All patients were followed-up at least until the end of their planned antitubercular treatment. - Table 1 shows important clinical, radiological, laboratory, and histopathological features of the included patients. The most common symptoms were abdominal discomfort in 88 (73.3\%; 95\% Cl 65.4-81.3\%) patients and fever in 30 (25.0\%; 95\% CI 17.3-32.8\%) patients. In premenopausal women menstrual irregularity was present in 10 (12.7\%; 95\% CI 5.3-19.9\%) patients. A past history of tuberculosis was elicited in 6 (5\%; 95\% CI 1.1-8.9\%) patients (pulmonary tuberculosis in 5 and spinal tuberculosis in 1 patient), and 12 (10.0\%; 95\% CI 5.3-16.8\%) patients had a history of abdominal surgery in the past.

On imaging studies, the common findings were ascites in 112 (93.3\%; 95\% CI 88.9-97.8\%) patients, adnexal enlargement in 63 (52.5\%; 95\% Cl 43.6-61.4\%) patients, and pleural effusion in 16 (13.3\%; 95\% CI 7.3-19.4\%) patients. Based on radiological findings, a diagnosis of tuberculosis over malignancy was favored in 19 (15.8\%; 95\% CI 9.8-23.6\%) patients.

Serum CA-125 level was obtained in all patients with a mean value of 666.9 (range, $38-18,554$ ) $\mathrm{U} / \mathrm{mL}$ and level more than $100 \mathrm{U} / \mathrm{mL}$ in 116 (96.7\%; 95\% CI 93.5-99.9\%) patients. Since patients were referred with a provisional diagnosis of intra-abdominal malignancy, other serum tumor markers like CEA and CA19.9 were also obtained in some patients. Mean CEA level was 1.7 (range, 0.2-8) $\mathrm{ng} / \mathrm{mL}$ and mean serum CA 19.9 level was 14.5 (range, $0-273) \mathrm{U} / \mathrm{mL}$.

Cytopathological examination of ascitic fluid was performed in all 112 patients with ascites and these samples were negative for malignant cells. The cytology smears showed lymphocyte rich exudate in 103 (91.9\%; 95\% Cl 86.9$97.0 \%)$ patients, granulomas in $2(1.8 \% ; 95 \% \mathrm{CI} 0.0-4.2 \%)$ patients, reactive mesothelial cells in 4 (3.6\%; $95 \%$ CI $0.1-$ $7.0 \%$ ) patients, and acute inflammatory cells in 3 (2.7\%; $95 \%$ CI 0.0-5.7\%) patients. Ascitic fluid ADA level was obtained in 112 (93.3\%) patients with mean level of 78.9 (range, 20-236) $\mathrm{U} / \mathrm{L}$. ADA level exceeding the upper limit of normal range (30 U/L) was seen in 109 (97.3\%; 95\% CI 94.3-100\%) patients, more than $40 \mathrm{U} / \mathrm{L}$ (suspicious for tuberculosis threshold in our laboratory) in 107 (95.5\%; 95\% CI 91.7-99.3\%) patients, and more than $60 \mathrm{U} / \mathrm{L}$ (threshold for high probability of tuberculosis in our laboratory) in 80 (71.4\%; 95\% CI 63.179.8\%) patients.

Ascitic fluid acid-fast (Ziehl-Neelsen [ZN]) staining was performed in 62 patients and was positive in only 4 (6.5\%; 95\% CI 0.3-12.6\%) patients while ascitic fluid culture examination for Mycobacterium tuberculosis, using LowensteinJensen medium, was performed in 59 patients, of whom it 
was positive in 7 (11.9\%; 95\% CI 3.6-20.1\%) patients, including two with positive ZN staining. Xpert MTB was done in 9 patients and was positive in 3 (33.3\%; 95\% CI $7.5-70.1 \%$ ) of them.

The diagnosis of peritoneal tuberculosis was established by histopathological examination of image-guided biopsy in $46(38.3 \%)$ patients and of surgical biopsy in $8(6.7 \%)$ patients while in remaining 66 (55\%) patients, peritoneal tuberculosis was the presumptive diagnosis based on a combination of clinical, radiological, and laboratory findings. The surgical biopsy was performed by laparoscopic approach in 2 (25\%) patients and by laparotomy in $6(75 \%)$ patients. All patients received standard antituberculosis treatment with isoniazid, rifampicin, pyrazinamide, and ethambutol for a duration of 9 months. However, treatment was extended to 12 months in $11(9.2 \%)$ patients based on partial response to 9 months of treatment. All patients with presumptive diagnosis of tuberculosis $(n=66)$ responded to antitubercular treatment and none of these patients was diagnosed to have malignancy during follow-up. At 2 months after starting antitubercular treatment serum CA-125 levels were available in 56 (46.7\%) patients with a mean value of $85.9 \mathrm{U} / \mathrm{mL}$ and range of 5.7-352 U/mL. Two (1.7\%) patients were diagnosed with multidrug-resistant (MDR) tuberculosis based on culture and drug sensitivity testing and treated accordingly. A total of 6 (5\%) patients died during follow-up.

\section{Discussion}

This study highlights the overlapping clinical, radiological, and laboratory characteristics of patients with peritoneal tuberculosis and those with advanced ovarian or primary peritoneal cancer which led to referral to our cancer center with provisional diagnoses of malignancy.

Peritoneal tuberculosis accounts for approximately 35\% of all cases of abdominal tuberculosis ${ }^{8}$ and is classified into three types based on clinical presentation, namely, wet, dry or plastic, and fibrotic-fixed. ${ }^{9}$ Wet type is the most common presentation of peritoneal tuberculosis and is characterized by abdominal pain, fever, and ascites. The dry type is characterized by peritoneal inflammation and adhesion formation secondary to caeseating nodules. The fibrotic-fixed type is the least common and these patients often present with subacute intestinal obstruction and omental masses. In our series an overwhelming majority (93.3\%) of patients had the wet-type presentation.

The symptoms and signs of both peritoneal tuberculosis and advanced ovarian cancer are often nonspecific including abdominal distension, discomfort or pain, breathlessness, weight loss, presence of ascites, and pelvic mass. Some studies have suggested that younger age at presentation (median 20-40 years) in patients with peritoneal tuberculosis could be a differentiating feature from malignancy (median age $50-70$ years). ${ }^{6}$ However, this distinction is less pronounced in our population, with median age of 41 years in the current series, while that of ovarian cancer patients being 52 years. ${ }^{10}$ Fever, which was present in almost one-fourth of patients, is a rare symptom in patients with ovarian cancer and should raise the suspicion of an infective pathology. Loss of appetite, menstrual irregularities, and past personal history of tuberculosis and family history of tuberculosis have been variably reported in patients with both peritoneal tuberculosis and malignancy and do not sufficiently discriminate between them.

Abdominal imaging in peritoneal tuberculosis often mimics malignancy and has been reported to have limited diagnostic accuracy. ${ }^{9,11}$ Ascites, peritoneal and omental disease, pelvic mass, and enlarged retroperitoneal lymph nodes are common in both tuberculosis and advanced malignancy. ${ }^{3}$ However, certain radiological features may favor tubercular pathology over malignancy and an experienced radiologist may be able to provide a differential diagnosis. On abdominal ultrasound, ascites with fine fibrous strands and lymphadenopathy with hypoechoic cores suggestive of caseation indicate a higher probability of tuberculosis. ${ }^{12}$ An abdominal contrast CT scan showing septate or particulate ascites; omental fat stranding; ill-defined adnexal masses; smooth, strongly enhancing peritoneal thickening; and caseous lymph nodes favor tuberculosis while well-defined, heterogeneous adnexal masses, nodular peritoneal thickening, and a nodular or caked omentum favor malignancy. ${ }^{13}$ In the current study peritoneal tuberculosis was suspected in only a minority (15.8\%) of patients based on imaging findings, which included one or more of the following features: presence of necrotic, calcified retroperitoneal lymph nodes; encysted fluid in the abdomen/pelvis; peritoneal thickening; clumping of ileal loops; mesenteric stranding; omental thickening without caking; ascites with internal echoes; ill-defined pelvic masses; and hydrosalpinx. PET-CT scan, performed in only 6 patients, suggested the diagnosis of advanced malignancy in all of them indicating that it can be unreliable in making this distinction.

This study reinforces the nonspecific nature of serum CA- $125^{7,8}$ with high levels, conventionally associated with advanced ovarian cancer, seen in the majority of patients. On the other hand, the cytological examination of the ascitic fluid was more informative with a large majority (95\%) of patients showing lymphocyte-rich exudate without malignant cells. As reported by others, ${ }^{14}$ our results indicate that AFB smear positivity by ZN staining, tubercular culture, and presence of granulomas are relatively insensitive for the diagnosis of peritoneal tuberculosis. Further, our data, in conformity with literature, suggest that the majority of patients with peritoneal tuberculosis have elevated levels of ascitic fluid ADA. ${ }^{15}$

Due to lack of specific features on preoperative assessment, the diagnosis of peritoneal tuberculosis is often made after surgery for suspected ovarian malignancy. However, in our series only 8 (6.7\%) patients underwent surgical biopsy while in the remaining patients diagnosis was made either on image guided biopsy or on the basis of clinical and laboratory findings.

Thus, the presence of lymphocyte-rich exudate in ascitic fluid examination, absence of malignant cells and an ADA level above the normal limit suggests the diagnosis of peritoneal tuberculosis with high sensitivity, even in the 
Table 1 Clinical and laboratory characteristics of patients

\begin{tabular}{|c|c|c|}
\hline & $\begin{array}{l}\text { Number }(N \\
=120)\end{array}$ & Percentage \\
\hline \multicolumn{3}{|l|}{ Symptoms } \\
\hline Abdominal pain & 88 & $73.3 \%$ \\
\hline Fever & 30 & $25.0 \%$ \\
\hline Weight loss & 15 & $12.5 \%$ \\
\hline Loss of appetite & 10 & $8.3 \%$ \\
\hline alrregular menstrual cycles & 10 & $12.7 . \%$ \\
\hline \multicolumn{3}{|l|}{ Menstrual status } \\
\hline Premenopausal & 79 & $65.8 \%$ \\
\hline Postmenopausal & 28 & $23.3 \%$ \\
\hline Not known & 13 & $10.8 \%$ \\
\hline \multicolumn{3}{|l|}{ Comorbidity } \\
\hline Diabetes mellitus & 2 & $1.6 \%$ \\
\hline Hypothyroidism & 4 & $3.3 \%$ \\
\hline Hypertension & 4 & $3.3 \%$ \\
\hline Chronic renal disease & 1 & $0.8 \%$ \\
\hline $\begin{array}{l}\text { Past history of cancer } \\
\text { (breast cancer, lymphoma, } \\
\text { and germ cell tumor, one } \\
\text { each) }\end{array}$ & 3 & $2.5 \%$ \\
\hline Abdominal surgery in past & 12 & $10.0 \%$ \\
\hline TB in past & 6 & $5.0 \%$ \\
\hline \multicolumn{3}{|l|}{$\begin{array}{l}\text { Imaging } \\
\text { (UGG/CE CT scan) }\end{array}$} \\
\hline Ascites & 112 & $93.3 \%$ \\
\hline Adnexal enlargement & 63 & $52.5 \%$ \\
\hline Omental involvement & 47 & $39.1 \%$ \\
\hline $\begin{array}{l}\text { Peritonealdeposits/ } \\
\text { thickening }\end{array}$ & 29 & $24.2 \%$ \\
\hline $\begin{array}{l}\text { Retroperitoneal lymph } \\
\text { nodes }\end{array}$ & 22 & $18.3 \%$ \\
\hline Pleural effusion & 16 & $13.3 \%$ \\
\hline Bowel-wall thickening & 11 & $9.2 \%$ \\
\hline Hepatosplenomegaly & 5 & $4.2 \%$ \\
\hline Mesenteric lymph node & 19 & $15.8 \%$ \\
\hline \multicolumn{3}{|l|}{ Ascitic fluid cytology $(N=112)$} \\
\hline Lymphocyte rich smear & 103 & $91.9 \%$ \\
\hline $\begin{array}{l}\text { Lymphocyte rich smear with } \\
\text { granuloma }\end{array}$ & 2 & $1.8 \%$ \\
\hline Reactive mesothelial cells & 4 & $3.6 \%$ \\
\hline Acute inflammatory smear & 3 & $2.7 \%$ \\
\hline $\begin{array}{l}\text { Ascitic fluid ZN staining positive } \\
(N=62)\end{array}$ & 4 & $6.5 \%$ \\
\hline Ascitic fluid AFB culture $(N=59)$ & 7 & $11.9 \%$ \\
\hline Xpert MTB $(N=9)$ & 3 & $33.3 \%$ \\
\hline \multicolumn{3}{|l|}{ Biopsy $(N=52)$} \\
\hline USG guided & 34 & $65.4 \%$ \\
\hline CT guided & 10 & $19.2 \%$ \\
\hline
\end{tabular}

\begin{tabular}{|l|l|l|}
\hline \multicolumn{1}{|c|}{ Surgical-laparoscopic } & 2 & $3.8 \%$ \\
\hline Laparotomy & 6 & $11.5 \%$ \\
\cline { 1 - 1 } Mean serum CA-125 $(N=120)$ & $666.9($ range, $38-18,554)$ \\
\cline { 1 - 1 } Mean serum CA19.9 $(N=88)$ & $14.5($ range, $0-273) \mathrm{U} / \mathrm{mL}$ \\
\cline { 1 - 1 } Mean serum CEA $(N=100)$ & 1.7 (range, $0.2-8) \mathrm{ng} / \mathrm{mL}$ \\
\cline { 1 - 1 } Mean ascitic fluid ADA $(N=112)$ & $78.9($ range, $20-236) \mathrm{U} / \mathrm{L}$ \\
\hline
\end{tabular}

Abbreviations: TB, tuberculosis; CECT, contrast enhanced computed tomography; ZN, Ziehl-Neelsen; AFB, acid-fast bacilli; USG, ultrasonography; CA, ... CA, cancer antigen; CEA, carcino embryonic antigen CEA, ...; ADA, adenosine deaminase.

${ }^{a}$ Calculated only in premenopausal women.

absence of bacteriological culture confirmation. Of note, no patient in our series who was started on tuberculosis treatment on the basis of clinical and laboratory findings turned out to have malignancy during follow-up. Our analysis also highlights the importance of obtaining a cytological or histological confirmation of malignancy before starting neoadjuvant chemotherapy in a patient with suspected advanced malignancy.

The strengths of the current study are a large sample size and uniformity of diagnostic and management protocol. The main limitations of our analysis include its retrospective nature and the possibility of not capturing the entire spectrum of patients with peritoneal tuberculosis because of being a cancer center. Diagnostic testing of tissue samples with Xpert was only done in a small minority of patients and could have increased the sensitivity of microbiological diagnosis.

\section{Conclusion}

Peritoneal tuberculosis in female patients can mimic advanced ovarian cancer or primary peritoneal carcinoma and should be included in the differential diagnosis, especially in developing countries, where tuberculosis remains endemic. Although bacteriological examination of ascitic fluid is insensitive, a combination of ascitic fluid cytological features and fluid ADA levels can aid in the diagnosis of tuberculosis in these patients. If these tests are negative in a patient with clinical suspicion of tuberculosis, image-guided/surgical biopsy using minimally invasive approach and frozen section examination should be planned, which helps to avoid unnecessary extensive surgery, related complications, and delay in starting tuberculosis treatment.

Authors' Contributions:

1. Amita Maheshwari-conceptualization, data curation, formal analysis, methodology, project administration, writing-original draft, writing-review and editing.

2. Sudeep Gupta-data curation, writing review, and editing.

3. Shweta Rai-data curation, writing review, and editing.

4. Bharat Rekhi-investigation, writing-review and editing.

5. Rohini Kelkar-investigation, writing-review and editing.

6. Shylasree TS-writing-review and editing. 
7. Santosh Menon-investigation, writing-review and editing.

8. Kedar Deodhar-investigation, writing-review and editing.

9. Meenakshi Thakur-investigation, writing-review and editing.

10. Ushasree Das-data curation.

11. Stuti Gupta-data curation.

12. Sandeep Tandon-methodology, writing-review and editing.

\section{Ethics Approval and Consent to Participate}

Institutional ethics committee approval (Number 3062) was sought and obtained for this analysis, with waiver of individual patient consent for this retrospective analysis.

\section{Conflict of Interest}

All authors declare no support from any organization for the submitted work, no financial relationships with any organizations that might have an interest in the submitted work, and no other relationships or activities that could appear to have influenced the submitted work.

\section{Funding}

There was no funding for this study.

\section{References}

1 Hopewell PC, Overview of clinical tuberculosis. In: Bloom BR, ed. Tuberculosis, Pathogenesis, Protection and Control. 1st ed. Washington, DC: American Society for Microbiology; 1994 25-46

2 Sinan T, Sheikh M, Ramadan S, Sahwney S, Behbehani A. CT features in abdominal tuberculosis: 20 years experience. BMC Med Imaging 2002;2(1):3
3 Koc S, Beydilli G, Tulunay G, et al. Peritoneal tuberculosis mimicking advanced ovarian cancer: a retrospective review of 22 cases. Gynecol Oncol 2006;103(2):565-569

4 Liu Q, Zhang Q, Guan Q, Xu JF, Shi QL. Abdominopelvic tuberculosis mimicking advanced ovarian cancer and pelvic inflammatory disease: a series of 28 female cases. Arch Gynecol Obstet 2014;289(3):623-629

5 Hu M-L, Lee C-H, Kuo C-M, et al. Abdominal tuberculosis: analysis of clinical features and outcome of adult patients in southern Taiwan. Chang Gung Med J 2009;32(5):509-516

6 Nayagam JS, Mullender C, Cosgrove C, Poullis A. Abdominal tuberculosis: diagnosis and demographics, a 10-year retrospective review from a single centre. World J Clin Cases 2016;4(8):207-212

7 Sharma JB, Jain SK, Pushparaj M, et al. Abdomino-peritoneal tuberculosis masquerading as ovarian cancer: a retrospective study of 26 cases. Arch Gynecol Obstet 2010;282(6):643-648

8 Uygur-Bayramicli O, Dabak G, Dabak R; Uygur-Bayrami \& Ccedil O. A clinical dilemma: abdominal tuberculosis. World J Gastroenterol 2003;9(5):1098-1101

9 Jadvar H, Mindelzun RE, Olcott EW, Levitt DB. Still the great mimicker: abdominal tuberculosis. AJR Am J Roentgenol 1997;168(6):1455-1460

10 Maheshwari A, Kumar N, Gupta S, et al. Outcomes of advanced epithelial ovarian cancer treated with neoadjuvant chemotherapy. Indian J Cancer 2018;55(1):50-54

11 Akhan O, Pringot J. Imaging of abdominal tuberculosis. Eur Radiol 2002;12(2):312-323

12 Kapoor VK. Abdominal tuberculosis. Postgrad Med J 1998;74(874):459-467

13 Rodríguez E, Pombo F. Peritoneal tuberculosis versus peritoneal carcinomatosis: distinction based on CT findings. J Comput Assist Tomogr 1996;20(2):269-272

14 Chaudery M, Mohamed F, Shirol S, Gudgeon M. An unusual presentation of intra-abdominal tuberculosis in a young man. J R Soc Med 2010;103(5):199-201

15 Riquelme A, Calvo M, Salech F, et al. Value of adenosine deaminase (ADA) in ascitic fluid for the diagnosis of tuberculous peritonitis: a meta-analysis. J Clin Gastroenterol 2006;40(8):705-710 